ESAIM: PROCEEDINGS, February 2007, Vol.16, 164-180

Eric Cancès \& Jean-Frédéric Gerbeau, Editors

DOI: $10.1051 /$ proc: 2007005

\title{
COHERENT VORTEX EXTRACTION IN 3D HOMOGENEOUS ISOTROPIC TURBULENCE USING ORTHOGONAL WAVELETS ${ }^{*, * *}$
}

\author{
Margarete O. Domingues ${ }^{1,4,2}$, Ingmar Broemstrup ${ }^{3}$, Kai Schneider $^{4,5}$, Marie \\ FARGE $^{1}$ AND BENJAMIN KADOCH ${ }^{4}$
}

\begin{abstract}
The coherent vortex extraction (CVE) decomposes each turbulent flow realization into two orthogonal components: a coherent and a random incoherent flow. They both contribute to all scales in the inertial range, but exhibit different statistical behaviour. The CVE decomposition is based on the nonlinear filtering of the vorticity field projected onto an orthonormal wavelet basis made of compactly supported functions. We decompose a 3D homogeneous isotropic turbulent flow at Taylor microscale Reynolds numbers $R_{\lambda}=140$ computed by a direct numerical simulation (DNS) at resolution $N=256^{3}$. Only $3.7 \% \mathrm{~N}$ wavelet modes correspond to the coherent flow made of vortex tubes, which contributes to $92 \%$ of the enstrophy. Another observation is that the coherent flow exhibits in the inertial range the same $k^{-5 / 3}$ slope in the energy spectrum and $k^{1 / 3}$ slope in the enstrophy spectrum as the total flow does. The remaining $96.3 \% N$ wavelet modes correspond to a random residual flow which is structureless, quasi equipartition of energy and a Gaussian velocity probability distribution function (PDF). We also analyse and visualize the Lamb vector, its divergence and curl and study the contributions coming from the coherent and incoherent components of vorticity and the induced velocity.
\end{abstract}

Résumé. L'extraction de tourbillons cohérents (Coherent Vortex Extraction: CVE) décompose un écoulement turbulent en deux composantes orthogonales: un écoulement cohérent et un écoulement aléatoire incohérent. Ces deux composantes contribuent aux grandes échelles dans la zone inertielle, mais ont des comportements statistiques différents. La décomposition CVE est basée sur le filtrage non linéaire du champ de vorticité projeté sur une base orthonormale d'ondelettes construite à partir de fonctions à support compact. On a décomposé un écoulement turbulent homogène isotrope 3D au nombre de Reynolds basé sur la microéchelle de Taylor $R_{\lambda}=140$ calculé par simulation numérique directe (DNS) à la résolution $N=256^{3}$. Seuls $3.7 \% N$ des modes d'ondelettes correspondent à l'écoulement cohérent construit à partir des tubes de vorticité, et contribuent à $92 \%$ de l'enstrophie. On peut observer également dans la zone inertielle, que l'écoulement cohérent présente une pente $k^{-5 / 3}$ pour son spectre d'énergie et $k^{1 / 3}$ pour son spectre d'enstrophie, similaires aux pentes trouvées pour l'écoulement total. Les $96.3 \% \mathrm{~N}$ modes d'ondelettes restants correspondent à un écoulement résiduel aléatoire qui ne présente aucune structure organisée, mais présente une quasi équipartition de l'énergie et une densité de probabilité (PDF) Gaussienne de la vitesse. On a également analysé et visualisé le vecteur de Lamb, sa divergence et son rotationnel, ainsi qu'étudié leurs différentes contributions en utilisant les parties cohérentes et incohérentes de la vorticité et de la vitesse induite.

* The authors thank the CIRM in Marseille for its hospitality and for financial support during the CEMRACS 2005 summerprogram where part of the work was carried out. ' $M F$ and $K S$ ' thankfully acknowledge financial support from the ANR project M2TFP.

** M.O. Domingues thankfully acknowledges financial support from the European Union project IHP on 'Breaking Complexity' (contract HPRN-CT 2002-00286).

${ }^{1}$ LMD-IPSL-CNRS, Ecole Normale Supérieure 24, rue Lhomond, 75231 Paris, Cedex 05, France

${ }^{2}$ Laboratório Associado de Computação e Matemática Aplicada (LAC), Instituto Nacional de Pesquisas Espaciais (INPE), Av. dos Astronautas, 1758, 12227-010 São José dos Campos, Brazil

3 Center for Multiscale Plasma Dynamics (CMPD), University of Maryland, College Park, MD 20742, USA

${ }^{4}$ Laboratoire de Modélisation et Simulation Numérique en Mécanique et Génie des Procédés (MSNM-GP), CNRS and Universités d'Aix-Marseille, 38, rue F. Joliot-Curie, 13451 Marseille Cedex 20, France

${ }^{5}$ Centre de Mathématiques et d'Informatique (CMI), Université de Provence, 39 rue F. Joliot-Curie, 13453 Marseille Cedex 13, France

e-mail: margarete@lac.inpe.br ibroemst@physics.umd.edu kschneid@cmi.univ-mrs.fr farge@lmd.ens.fr kadoch@l3m.univ-mrs.fr

(c) EDP Sciences, SMAI 2007 


\section{Contents}

1. Introduction

2. Coherent Vortex Extraction (CVE)

2.1. Orthogonal wavelets

2.2. Algorithm for extraction

3. Application to 3D turbulence

3.1. Homogeneous isotropic flow

3.2. Vorticity and velocity

\section{INTRODUCTION}

Turbulent flows typically exhibit well organized structures evolving in a random background. Since these organized structures are well localized and excited on a wide range of scales, the wavelet representation to analyze such flows was proposed in [4]. Wavelet bases are well suited for this task, because there are made of self-similar functions well localized in both physical and spectral spaces leading to an efficient hierarchical representation of intermittent data, as encounted in turbulent flows [4].

In different papers, $[5,7,8,12]$, the extraction of coherent vortices out of turbulent flows using the wavelet techniques is introduced. The focus of the method is not on the coherent structures themselves, but on the noise: coherent structures are by definition what remains after the denoising, while the noise is supposed to be Gaussian and decorrelated. The coherent vortex extraction (CVE) method is based on a wavelet decomposition of the vorticity field, a subsequent thresholding of the wavelet coefficients and a reconstruction from those coefficients whose modulus is above a given threshold. The value of the threshold is based on mathematical theorems yielding an optimal min-max estimator for the denoising of intermittent data $[2,3]$.

In [7] the vortex extraction technique was introduced for two-dimensional flows using scalar-valued wavelet decompositions. In [5] this technique was extended to three-dimensional flows using a vector-valued wavelet decomposition and in [11] orthogonal and biorthogonal wavelets are compared. In these papers for 2D and 3D turbulence, respectively, it has been shown that a few strong wavelet coefficients represent the organized part of the flow, i.e, the coherent vortices, while the remaining weak coefficients represent the incoherent background flow which is structureless and noise like.

The aim for the present paper is to study the coherent vortex extraction and to analyze the Lamb vector for the filtered flows. The Lamb vector, its divergence and also its curl are of interest for aeroacustics and turbulence modelling using the Coherent Vortex Simulations (CVS) approach.

This paper is organized as follows. In section 2 we recall the CVE algorithm. In Section 3 we present results of the CVE applied to DNS data of homogeneous isotropic turbulence for Taylor microscale based Reynolds numbers of 140. We also analyze the Lamb vector, its divergence and its curl. Section 4 presents the conclusions of the paper.

\section{Coherent Vortex Extraction (CVE)}

\subsection{Orthogonal wavelets}

For the CVE decomposition we use an orthogonal multiresolution analysis (MRA). The three-dimensional MRA of $L^{2}\left(R^{3}\right)$ is obtained through the tensor product of three one-dimensional MRA's of $L^{2}(R)$. In this context a function $f \in L^{2}\left(R^{3}\right)$ can be developed into a three-dimensional wavelet basis

$$
f(\vec{x})=\sum_{\gamma \in \Gamma} \widetilde{f}_{\gamma} \psi_{\gamma}(\vec{x}),
$$

where the multiindex $\gamma=\left(j, i_{x}, i_{y}, i_{z}, \mu\right)$ denotes the scale $j$, the positions $\vec{i}=\left(i_{x}, i_{y}, i_{z}\right)$ and the seven directions $\mu=1, \ldots, 7$ of the wavelets. The index set $\Gamma$ is

$$
\Gamma=\left\{\gamma=\left(j, i_{x}, i_{y}, i_{z}, \mu\right) \quad j=0, \ldots, J-1 \quad i_{x}, i_{y}, i_{z}=0, \ldots, 2^{j}-1 \quad \mu=1, \ldots, 7\right\}
$$


Due to orthogonality the wavelet coefficients are given by $\widetilde{f}_{\gamma}=\left\langle f, \psi_{\gamma}\right\rangle$ where $\langle\cdot, \cdot\rangle$ denotes the $L^{2}$ inner product. For more details on this construction and in wavelets we refer the reader to the standart textbook [1] and also to the article [4].

The Coifman 12 wavelets are chosen here because they are almost symmetric and they have compact support [1]. Futhermore, they have $M=4$ vanishing moments and the corresponding quadratic mirror filter has a length of $3 M=12[1]$.

\subsection{Algorithm for extraction}

We consider a $3 \mathrm{D}$ vorticity $\vec{\omega}=\vec{\omega}(x, y, z)$ field $\vec{\omega}=\nabla \times \vec{v}$, where $\vec{v}=\vec{v}(x, y, z)$ is the velocity field. The three components of $\vec{\omega}$ are developed into an orthonormal wavelet series, from the largest scale $l_{\text {max }}=2^{0}$ to the smallest scale $l_{\min }=2^{-J+1}$, using a $3 \mathrm{D}$ MRA as presented in [4].

The vorticity field is decomposed into coherent vorticity $\vec{\omega}_{c}=\vec{\omega}_{c}(x, y, z)$ and incoherent vorticity $\vec{\omega}_{i}=\vec{\omega}_{i}(x, y, z)$ by projecting its three components onto an orthonormal wavelet basis and applying nonlinear thresholding to the wavelet coefficients. The choice of the threshold is based on theorems $[2,3]$ proving optimality of the wavelet representation for denoising signals - optimality in the sense that wavelet-based estimators minimize the maximum $L^{2}$-error for functions with inhomogeneous regularity in the presence of Gaussian white noise. We have chosen the variance of the total vorticity instead of the variance of the noise, which gives the threshold $T=\left(\frac{4}{3} Z \log N\right)^{\frac{1}{2}}$, where $Z=\frac{1}{2}\langle\vec{\omega}, \vec{\omega}\rangle$ is the total enstrophy (which is half the variance) and $N^{3}$ is the resolution. Notice that this threshold does not require any adjustable parameters.

In summary, we compute the modulus of the wavelet coefficients:

$$
|\overrightarrow{\tilde{\omega}}|=\left|\overrightarrow{\tilde{\omega}}_{\gamma}\right|=\left(\sum_{n=1}^{3}\left[\tilde{\omega}_{\gamma}\right]_{n}^{2}\right)^{\frac{1}{2}} .
$$

Then, the coherent vorticity is reconstructed from the wavelet coefficients whose modulus is larger than the threshold $T$, while the incoherent vorticity is computed by the difference with the total field. The two fields thus obtained, $\vec{\omega}_{c}$ and $\vec{\omega}_{i}$, are orthogonal, which ensures the decomposition of the total enstrophy into $Z=Z_{c}+Z_{i}$.

The CVE decomposition algorithm consists of three fast wavelet transforms (WT) for each vorticity component, a thresholding of the wavelet coefficients and three inverse fast wavelet transforms (IWT), one for each component of the $\overrightarrow{\tilde{\omega}}_{c}$, i.e., all coefficient with $|\overrightarrow{\tilde{\omega}}|$ greater than the threshold, form the coherent vorticity $\left(\vec{\omega}_{c}\right)$. The incoherent vorticity $\vec{\omega}_{i}$ components are in principle computed using the inverse wavelet transform from the weak coefficients. In order to simplify computations we performed the difference between total and coherent vorticity which yields the same result. A flowchart of the CVE algorithm is depicted in Fig. 1. The induced coherent and incoherent velocity fields are computed using Biot-Savat's law (BS), $\vec{v}=\nabla \times\left(\nabla^{-2} \vec{\omega}\right)$, from the coherent and incoherent vorticity fields, respectively. The computational cost of the fast wavelet transform is of order $C N$, where $N$ is the resolution, and $C$ is proportional to the filter length. Therefore the total number of operations is $O(N)$, while it is $O\left(N \log _{2} N\right)$ for the Fast Fourier Transform (FFT) [6]. For BS the FFT algorithm is also used.

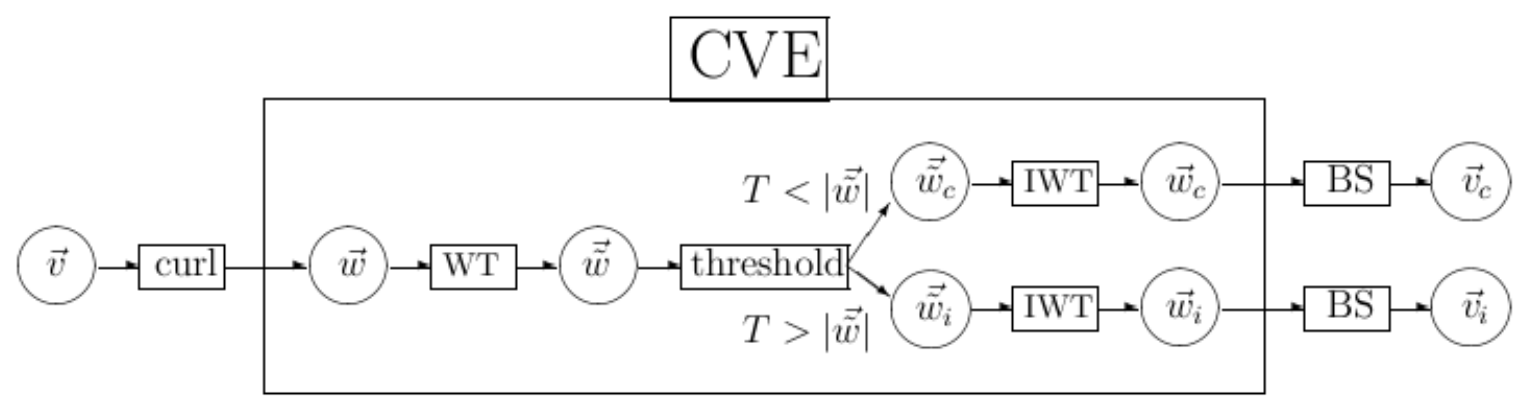

Figure 1. Flowchart of CVE decomposition. 


\section{Applichtion to 3D turbulence}

\subsection{Homogeneous isotropic flow}

The data to be analyzed corresponds to homogeneous isotropic turbulence, with a stochastic forcing at large scales, kindly provided by P.K. Yeung and his group from Georgia Tech. The fields are computed at resolution $N=2^{3 J}$, where $N$ is the number of grid points and $J$ the number of octaves in each direction. We are analyzing fields which have been computed at resolutions of $N=256^{3}$ which corresponds to microscale based Reynolds numbers $R_{\lambda}=140$. More details about these simulations can be found in [13]. We study the velocity field reconstructed by BS from the vorticity field.

\subsection{Vorticity and velocity}

In Table 1 the statistical properties of the vorticity and velocity are presented. We observe that the coherent part of the vorticity corresponds to $3.6 \%$ of the wavelet coefficients, which maintain $92.5 \%$ of the total enstrophy and $99.7 \%$ of the total energy. The corresponding velocity fields are reconstructed by Biot-Savart's relation from the vorticity and its coherent and incoherent parts. As wavelets are 'almost' eigenfunctions of the CalderonZygmund singular kernels [10], such as BS kernel, the coherent and incoherent velocity fields are only quasiorthogonal and the total energy is decomposed into $E=E_{c}+E_{i}+\varepsilon$, where $E=\frac{1}{2}\langle\vec{v}, \vec{v}\rangle$ and $\varepsilon=0.18 \% E$ in this case.

\begin{tabular}{lccc}
\hline \hline \multicolumn{4}{c}{ Field with } \\
& $R_{\lambda}=140$ & \\
& total & coherent & incoherent \\
\hline \% of coefficients & 100 & 3.59 & 96.41 \\
\hline Vorticity & & & \\
\hline Enstrophy & 212.80 & 196.93 & 15.87 \\
\% of enstrophy & 100 & 92.54 & 7.46 \\
min value & -230.26 & -243.29 & -34.18 \\
max value & 245.86 & 259.24 & 38.19 \\
Vorticity skewness & -0.013 & -0.016 & $0.68 \cdot 10^{-3}$ \\
Vorticity flatness & 8.08 & 8.31 & 4.92 \\
\hline Velocity & & & \\
\hline Energy & 2.955 & 2.946 & 0.004 \\
\% of energy & 100 & 99.7 & 0.12 \\
min value & -5.92 & -5.93 & -0.31 \\
max value & 6.16 & 6.17 & 0.33 \\
skewness & 0.1432 & 0.1439 & 0.0016 \\
flatness & 2.7818 & 2.7802 & 3.48 \\
\hline \hline
\end{tabular}

TABLE 1. Decomposition of the flow field of size $N=256^{3}$ corresponding to Reynolds number $R_{\lambda}=140$, using a Donoho threshold of 29.87 for the vorticity field.

A visualization of the modulus of vorticity and velocity and their coherent and incoherent fields are shown in Figure 2 and Figure 3, respectively. We observe that almost all structures are preserved in the coherent part and they are no organized structures left in the incoherent part. The velocity field is smoother than the vorticity field with less localized structures. Also for the incoherent part of the velocity field it is difficult to identify organized structures. The velocity and its coherent part exhibit very similar behaviours in the visualization what confirms the energy result shown in Table 1 . This can also be observed in the one dimensional cuts in the $x$-direction for the corresponding velocity fields, shown in Figure 4(a). On the other hand, the 1D cuts of vorticity show that the coherent part approximates well the total vorticity. However some peaks are over estimated. This confirms that slightly less enstrophy (92.5\%) is retained compared to energy (99.7\%) as shown in Table 1.

In Figure 5 vorticity and velocity PDFs are plotted for the total, coherent and incoherent parts of the field. We find that the PDFs of the total and coherent fields almost coincide, exibiting a Gaussian shape for the velocity with flatness 3 and a stretched exponential behaviour for vorticity with flatness about 8 . The incoherent parts show a strongly reduced variance with also a Gaussian shape (with flatness 3.48) for velocity while the vorticity PDF has exponential tails (with flatness 4.92). 
(a) $|\vec{\omega}|$

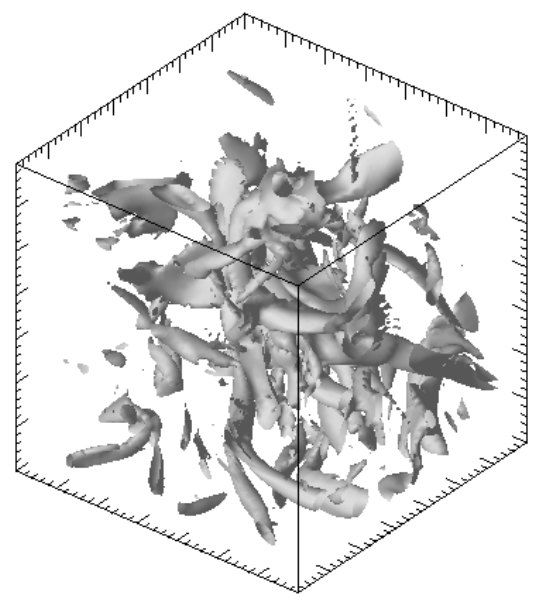

(b) $\left|\vec{\omega}_{c}\right|$

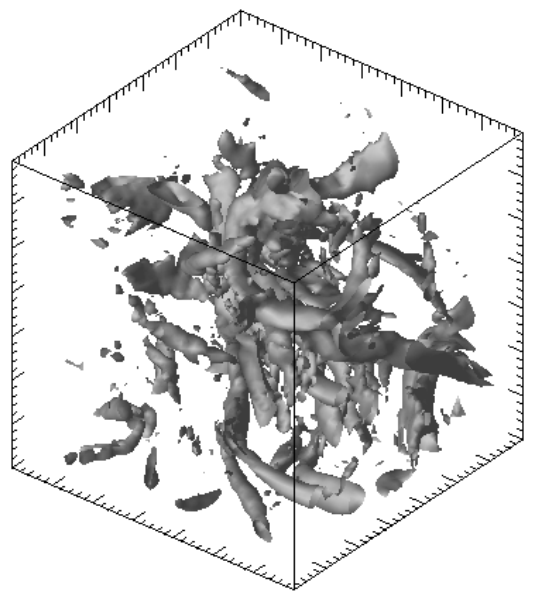

(c) $\left|\vec{\omega}_{i}\right|$

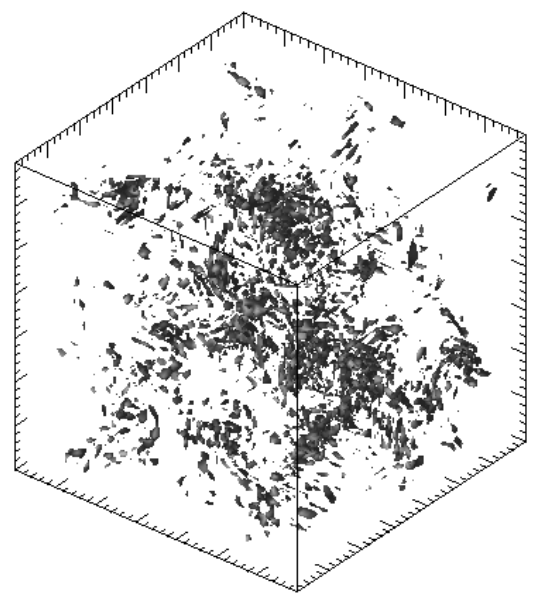

Figure 2. Modulus of vorticity, coherent and incoherent fields for $R_{\lambda}=140$. Only subcubes of the size $64^{3}$ out of the $256^{3}$ data sets are visualized to zoom in on the structures. The isosurfaces are $|\vec{\omega}|=3 \sigma^{s u b}$ for total and coherent parts and the standart deviation of the subcube $\sigma^{s u b}$, for the incoherent field with the standart deviation of the subcube $\sigma^{s u b}=13.58$.

In Figure 6 the enstrophy and energy spectra for the total, coherent and incoherent part of the fields are described. In this figure we observe that in the inertial range the coherent parts present a similar energy and enstrophy spectra compared to the spectra of the total field, whereas they differ only in the dissipative range for $k \geq 30$. 
(a) $|\vec{v}|$

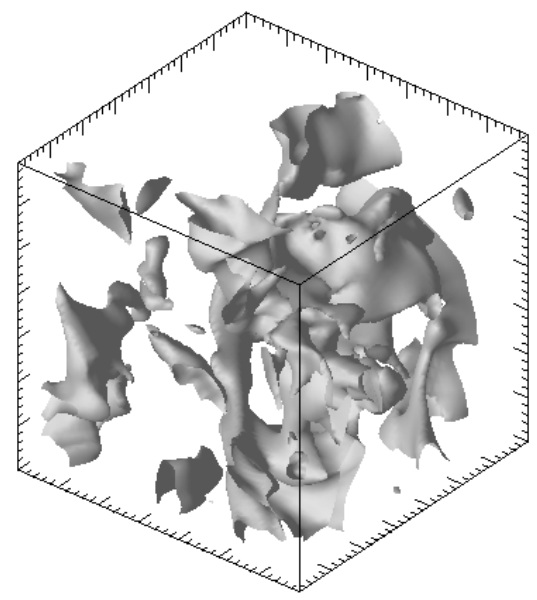

(b) $\left|\vec{v}_{c}\right|$

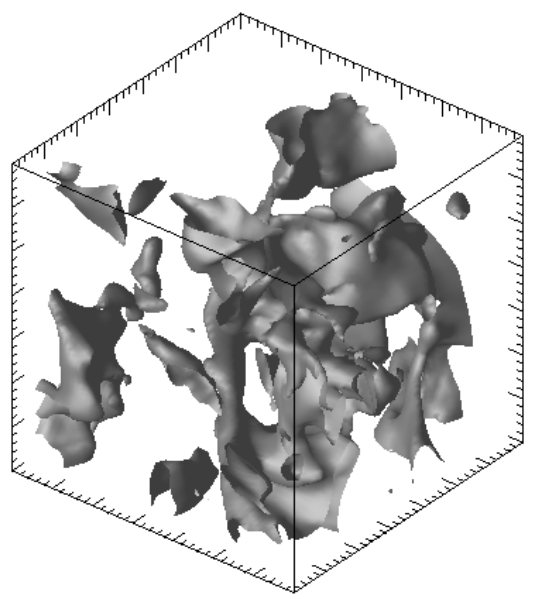

(c) $\left|\vec{v}_{i}\right|$

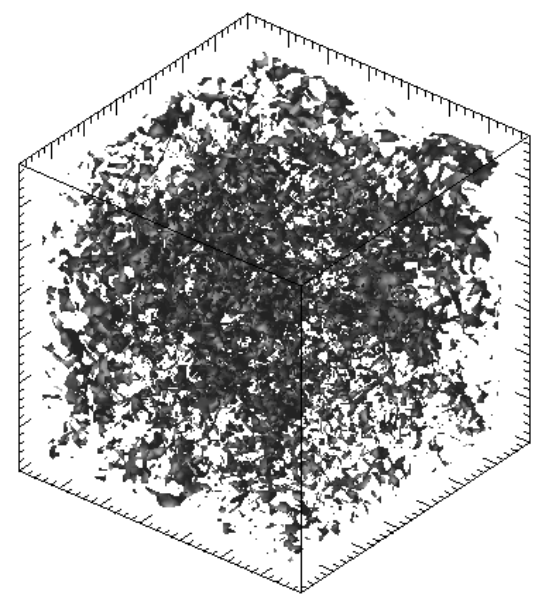

FiguRE 3. Modulus of velocity, coherent and incoherent fields for $R_{\lambda}=140$. Only subcubes $64^{3}$ out of $256^{3}$ data set are visualized to zoom in on the structures. The isosurfaces are $|\vec{v}|=3 \sigma^{\text {sub }}$ for total and coherent field and $\sigma^{s u b} / 10$, for the incoherent field with the standart deviation of the subcube $\sigma^{s u b}=0.79$. 
(a) $|\vec{\omega}|$

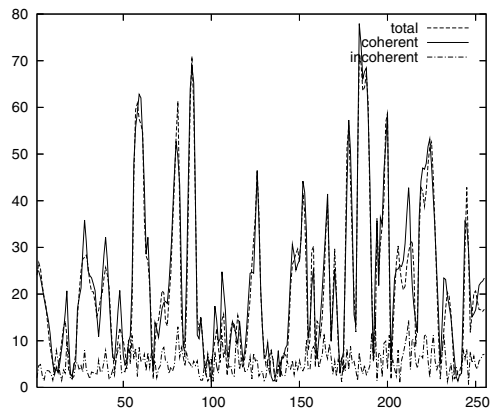

(b) $|\vec{v}|$

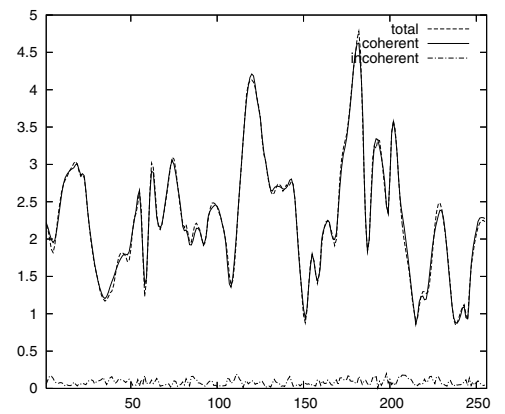

Figure 4. One dimensional cuts in $x$-direction, for $y=z=64 \pi / 256$, modulus of vorticity (a) and velocity (b) total field, coherent and incoherent fields for $R_{\lambda}=140$.

(a) PDF of $\vec{\omega}$

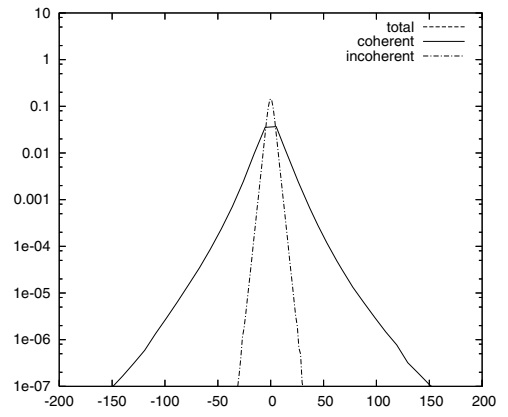

(b) PDF of $\vec{v}$

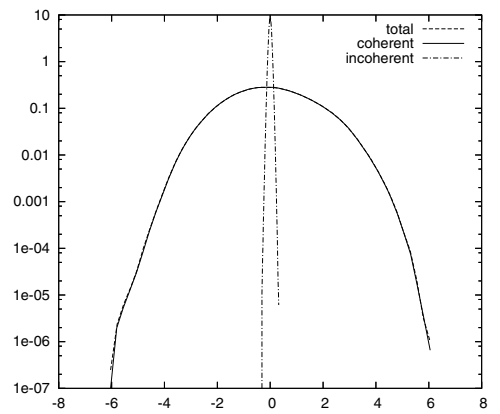

Figure 5. PDF of the modulus of vorticity (a) and velocity (b) total field, coherent and incoherent fields.

(a) spectrum of enstrophy

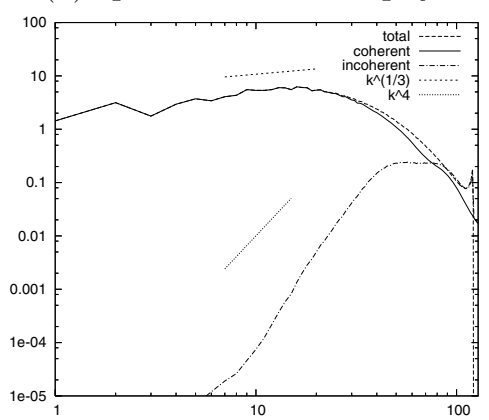

(b) spectrum of energy

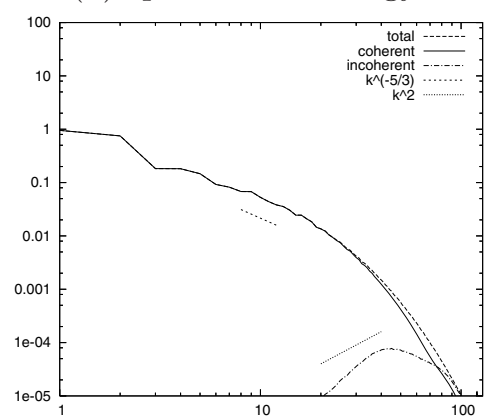

Figure 6. Entrophy (a) and (b) energy spectrum for the total field, coherent and incoherent fields. 


\subsection{Lamb vector, its divergence and its curl}

With the vorticity and the velocity fields we compute the Lamb vector $\vec{\ell}=\vec{\omega} \times \vec{v}$ and its variance $\Lambda=\frac{1}{2}<\vec{\ell}, \vec{\ell}>$, where the $\langle\vec{\imath}, \vec{\cdot}\rangle$ is the inner product for vector valued functions. The Lamb vector is used to analyse the influence of the coherent and incoherent parts of the nonlinear terms of the Euler equations

$$
\begin{aligned}
\frac{\partial \vec{v}}{\partial t}-\vec{\ell} & =\nabla p+\frac{1}{2} v^{2} \\
\vec{\nabla} \cdot \vec{v} & =0 .
\end{aligned}
$$

For this we propose to split the Lamb vector into four contributions based on the coherent and incoherent velocity and vorticity fields: $\vec{\ell}_{c c}=\vec{\omega}_{c} \times \vec{v}_{c}, \vec{\ell}_{i c}=\vec{\omega}_{i} \times \vec{v}_{c}, \vec{\ell}_{c i}=\vec{\omega}_{c} \times \vec{v}_{i}$ and $\vec{\ell}_{i i}=\vec{\omega}_{i} \times \vec{v}_{i}$. We define the corresponding variances as

$$
\Lambda_{c c}=\frac{1}{2}<\vec{\ell}_{c c}, \vec{\ell}_{c c}>, \quad \Lambda_{i c}=\frac{1}{2}<\vec{\ell}_{i c}, \vec{\ell}_{i c}>, \quad \Lambda_{c i}=\frac{1}{2}<\vec{\ell}_{c i}, \vec{\ell}_{c i}>, \quad \Lambda_{i i}=\frac{1}{2}<\vec{\ell}_{i i}, \vec{\ell}_{i i}>
$$

where $\Lambda=\Lambda_{c c}+\Lambda_{c i}+\Lambda_{i c}+\Lambda_{i i}$.

Table 2 shows the different contributions to the variance $\Lambda$ of the Lamb vector $\vec{\ell}$. As expected the component $\Lambda_{c c}$ is the largest contribution and retains $92 \%$ of the total contribution $\Lambda$. Consequentely, the other components, $\Lambda_{c i}, \Lambda_{i c}$ and $\Lambda_{i i}$, represent less then $8 \%$ of $\Lambda$, in which the $\Lambda_{i i}$ contribution is quasi zero $(0.01 \%)$.

\begin{tabular}{lrr}
\hline \hline & Value & $\%$ \\
\hline$\Lambda$ & 820.83 & 100 \\
\hline$\Lambda_{c c}$ & 756.97 & 92.22 \\
$\Lambda_{c i}$ & 1.17 & 0.14 \\
$\Lambda_{i c}$ & 62.66 & 7.63 \\
$\Lambda_{i i}$ & 0.09 & 0.01 \\
$\sum$ & & 100 \\
\hline \hline
\end{tabular}

TABLE 2. Different contributions to the variance $\Lambda$ of the Lamb vector $\vec{\ell}$.

In Figure 7 the 3D visualization of the four coherent and incoherent contributions of the Lamb vector are shown. Almost all structures are preserved in the visualization of the coherent contribution $\left|\vec{\ell}_{c c}\right|$. Some structures are also observed in the incoherent vorticity contribution $\left|\vec{\ell}_{i c}\right|$, and no organized contributions appear in $\left|\vec{\ell}_{i i}\right|$ and $\left|\vec{\ell}_{c i}\right|$. This suggested that the nonlinearity of the flow motion is preserved by the coherent part only. The PDF and the spectrum of the Lamb vector and the four contributions $\vec{\ell}_{c c}, \vec{\ell}_{c i}, \vec{\ell}_{i c}, \vec{\ell}_{i i}$ are exhibited in Figure 10 (a) and (b), respectively.

For aeroacustics, the divergence of the Lamb vector $(\nabla \cdot \vec{\ell})$ is a quantity of interest [9], thus we compute it. Following the previous studies, we compute the divergence of the four contributions of the Lamb vector, based on the coherent and incoherent velocity and vorticity. Again almost all structures are preserved in the coherent contribution $\nabla \cdot \vec{\ell}_{c c}$, as can be observed in Figure 8 . Some structures are also found in the incoherent vorticity contribution $\nabla \cdot \vec{\ell}_{i c}$, and no organized contributions appear in $\nabla \cdot \vec{\ell}_{i i}$ and $\nabla \cdot \vec{\ell}_{c i}$ fields. In Figure 11 the PDF and the spectrum of the divergence of the Lamb vector and the four contributions are plotted. In the spectrum we notice that the $\nabla \cdot \vec{\ell}_{c c}$ contribution and the divergence of the field are almost the same in the inertial range. They have also similar PDFs, skewness and flatness.

The variance of the divergence of the Lamb vector is defined as $\Delta=\frac{1}{2}\langle\nabla \cdot \vec{\ell}, \nabla \cdot \vec{\ell}\rangle$ and similarly we define the variances of the coherent and incoherent contributions as $\Delta_{c c}, \Delta_{c i}, \Delta_{i c}$ and $\Delta_{i i}$.

Table 3, presents the different contributions to the variance $\Delta$ of the divergence of the Lamb vector $\vec{\ell}$. The component $\Delta_{c c}$ is the largest contribution and retains $75 \%$ of the total contribution $\Delta$ and $\Delta_{i c}$ retains $21 \%$. The other components, $\Delta_{c i}$ and $\Delta_{i i}$, represent $0.5 \%$ of $\Delta$, and hence are quasi nonsignificant. These four contributions do not add up to $100 \%$, ie., there is a lost in the variance of $3.8 \%$. 
(a) $\left|\vec{\ell}_{c c}\right|$

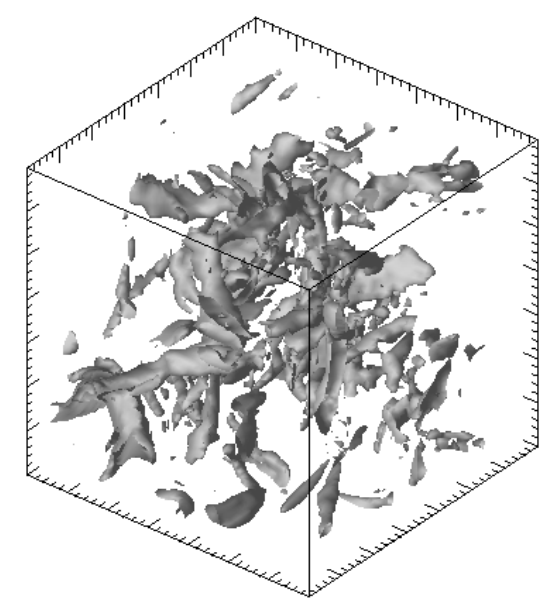

(c) $\left|\vec{\ell}_{i c}\right|$

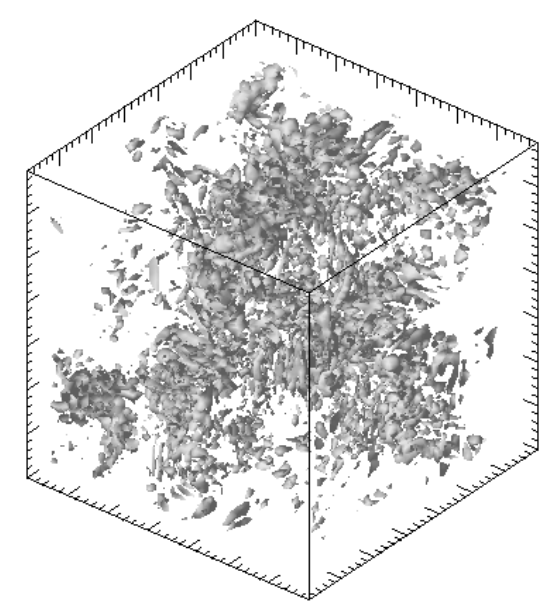

(b) $\left|\vec{\ell}_{c i}\right|$

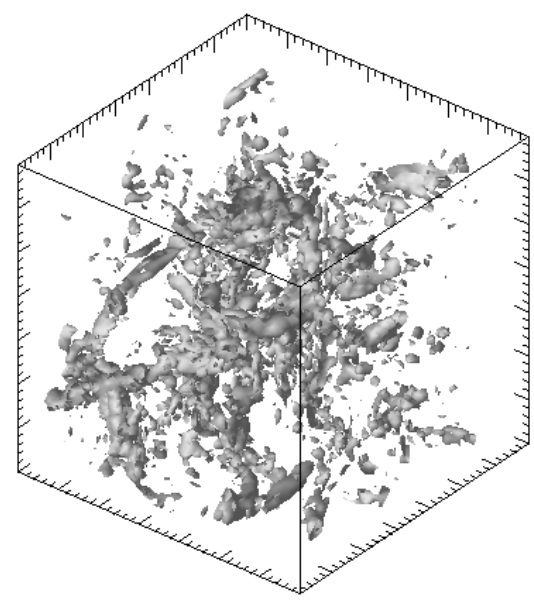

(d) $\left|\vec{\ell}_{i i}\right|$

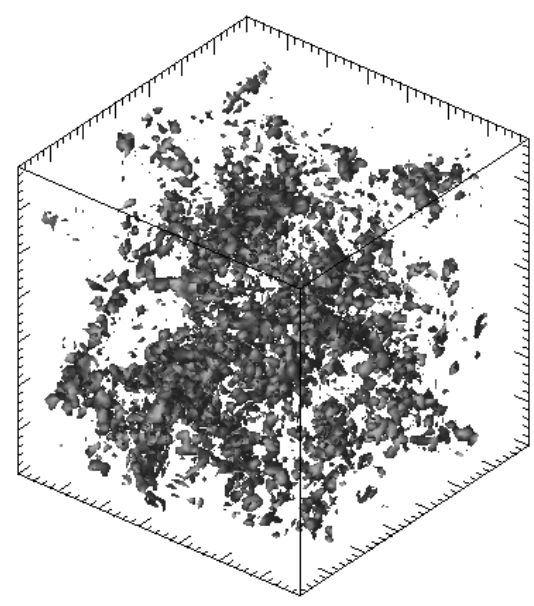

FiguRE 7 . The coherent and incoherent contributions of the Lamb vector for $R_{\lambda}=140$. Only subcubes $64^{3}$ are visualized to zoom in on the structures. The isosurface are $3 \sigma^{\text {sub }}$, where $\sigma^{\text {sub }}$ is the standart deviation of the subcube for each contribution: $\sigma^{s u b}=26.33$ (a), $\sigma^{\text {sub }}=1.3(\mathrm{~b})$, $\sigma^{s u b}=7.5(\mathrm{c})$, and $\sigma^{s u b}=0.33$. 
(a) $\nabla \cdot \vec{\ell}_{c c}$

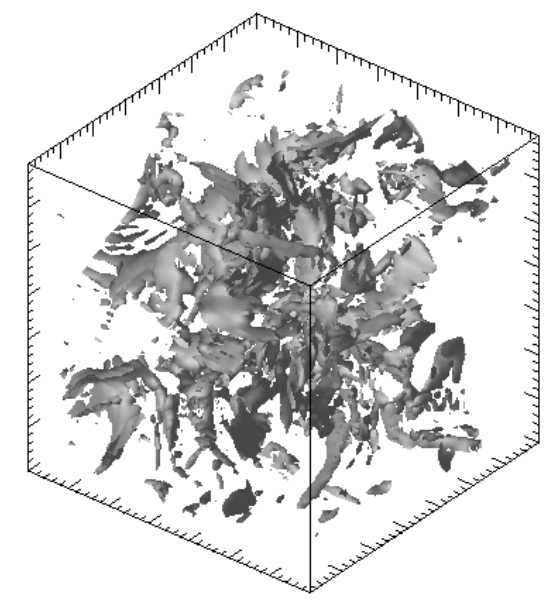

(c) $\nabla \cdot \vec{\ell}_{i c}$

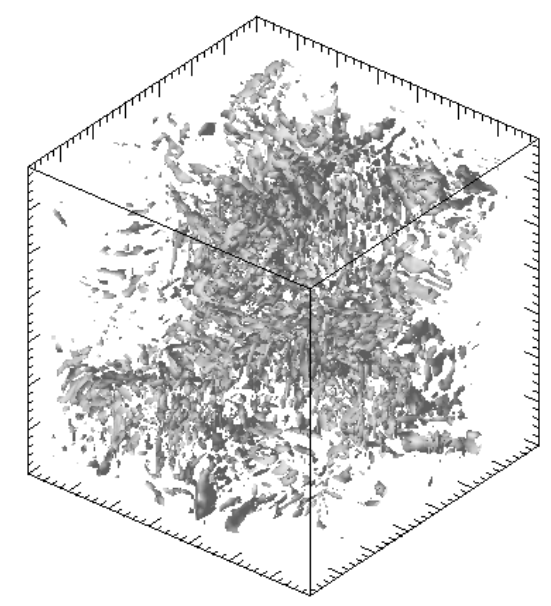

(b) $\nabla \cdot \vec{\ell}_{c i}$

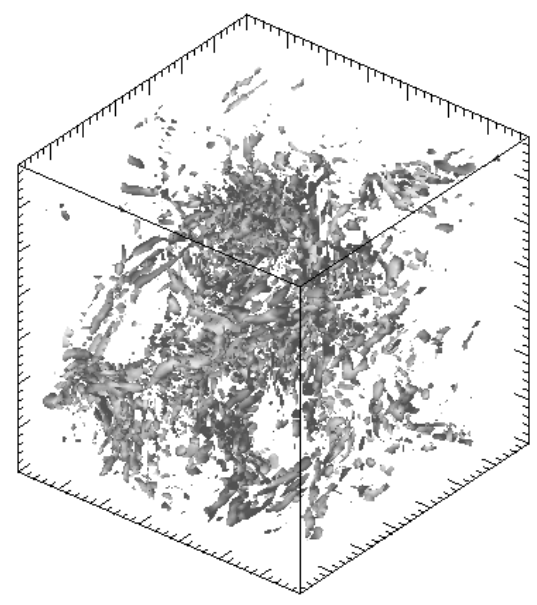

(d) $\nabla \cdot \vec{\ell}_{i i}$

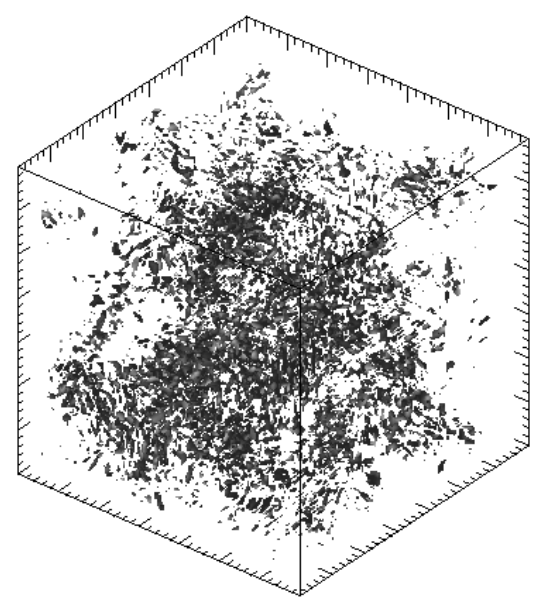

FiguRE 8. Components of the divergence of the Lamb vector for $R_{\lambda}=140$. The isosurfaces are $1.5 \sigma$ where $\sigma=1258.65$ for (a), 112.18 for (b) 689.14 for (c) and 41.21 for (d). 


\begin{tabular}{lrr}
\hline \hline & Value & $\%$ \\
\hline$\Delta$ & 888720.14 & 100 \\
$\Delta_{c c}$ & 667163.68 & 75.07 \\
$\Delta_{c i}$ & 4455.94 & 0.50 \\
$\Delta_{i c}$ & 183302.42 & 20.63 \\
$\Delta_{i i}$ & 641.89 & 0.00 \\
$\sum$ & & 96.20 \\
\hline \hline
\end{tabular}

TABLe 3. Different contributions to the variance $\Delta$ of the divergence of Lamb vector $\nabla \cdot \vec{\ell}$.

The curl of the Lamb vector $\nabla \times \vec{\ell}$ is the quantity of interest for studying the nonlinearity of the Navier-Stokes Equations without dissipation (also called Euler Equations), because we can write these equations as following:

$$
\begin{aligned}
\frac{\partial \vec{\omega}}{\partial t}-\vec{\nabla} \times \vec{\ell} & =\overrightarrow{0} \\
\vec{\nabla} \cdot \vec{v} & =0 .
\end{aligned}
$$

where the curl of the Lamb vector represents all the nonlinear behaviour of this set of equations.

In this analysis we compute the curl of the four contributions to the Lamb vector, based on the coherent and incoherent velocity and vorticity. The majority of structures is preserved in the visualization of the coherent contribution $\nabla \times \vec{\ell}_{c c}$. Some structures are also found in the incoherent vorticity contribution $\nabla \times \vec{\ell}_{i c}$, and no organized contribution appears in the $\nabla \times \vec{\ell}_{i i}$ and $\nabla \times \vec{\ell}_{c i}$ fields. This illustrates the importance of the coherent contributions for the nonlinear flow motion preservation.

In Figure 12 the PDF and the spectra of the curl of the Lamb vector and the four contributions are plotted. It is possible to see in these PDFs that the $\nabla \times \vec{\ell}_{c c}$ coherent contributions, the curl of the coherent part of the Lamb vector and of the total Lamb vector are very similar. It is also possible to observe in the spectrum that the $\nabla \times \vec{\ell}_{c c}$ contribution and the total field are almost the same in the inertial range.

To quantify $\nabla \times \vec{\ell}$ the variance $\Omega$ is defined as $\Omega=\frac{1}{2}\langle\nabla \times \vec{\ell}, \nabla \times \vec{\ell}\rangle$. Similary, for the coherent and incoherent contributions the sub indexes $c c, c i, i c, i i$ are used. Table 4 presents the different contributions to the variance $\Omega$ of the curl of the Lamb vector. The component $\Omega_{c c}$ is the largest contribution and it retains $92 \%$ of the total $\Omega$. Consequently, the other components, $\Omega_{c i}, \Omega_{i c}$ and $\Omega_{i i}$, represent less then $6 \%$ of $\Omega$. The $\Omega_{i i}$ contribution is quasi nonsignificant (0.1\%). The sum of these four contributions does not reach $100 \%$, it represents a loss of $2.4 \%$ of the variance.

\begin{tabular}{lrr}
\hline \hline & Value & $\%$ \\
\hline$\Omega$ & 374206.10 & 100 \\
\hline$\Omega_{c c}$ & 342956.78 & 91.65 \\
$\Omega_{c i}$ & 2084.35 & 0.56 \\
$\Omega_{i c}$ & 196970.93 & 5.26 \\
$\Omega_{i i}$ & 379.24 & 0.10 \\
$\sum$ & & 97.57 \\
\hline \hline
\end{tabular}

TABLE 4. Different contributions to the variance $\Omega$ of the curl of Lamb vector $\nabla \times \vec{\ell}$.

\section{Conclusions}

We have shown that the CVE method is an efficient tool for extracting coherent vortices out of turbulent flows. We have applied it to a 3D homogeneous isotropic turbulent flow. We decomposed this flow into their coherent parts, made of vortex tubes which interact nonlinearly and into an incoherent random background flow which is structureless and decorrelated. We also found that the incoherent velocity has a Gaussian PDF.

We analyzed in addition the Lamb vector, its divergence and its curl. The results show that the Lamb vector, its divergence and its curl exhibit organized structures. We applied the CVE algorithm to these fields and found 
(a) $\left|\nabla \times \vec{\ell}_{c c}\right|$

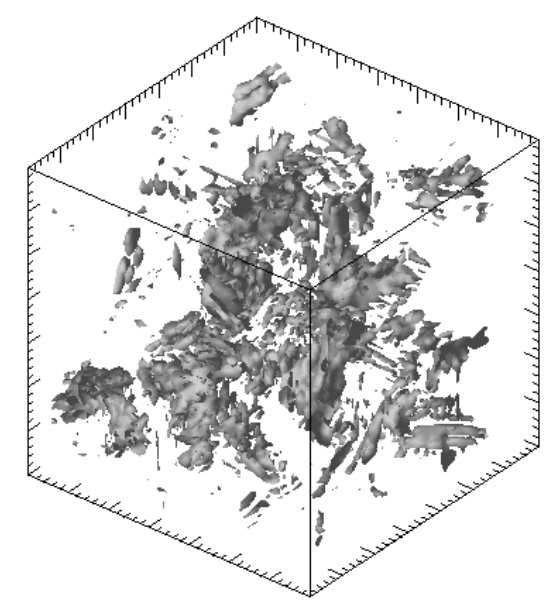

(c) $\left|\nabla \times \vec{\ell}_{i c}\right|$

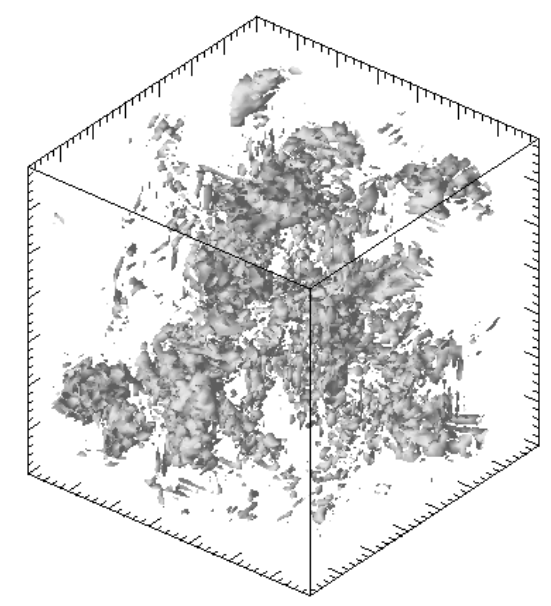

(b) $\left|\nabla \times \vec{\ell}_{c i}\right|$

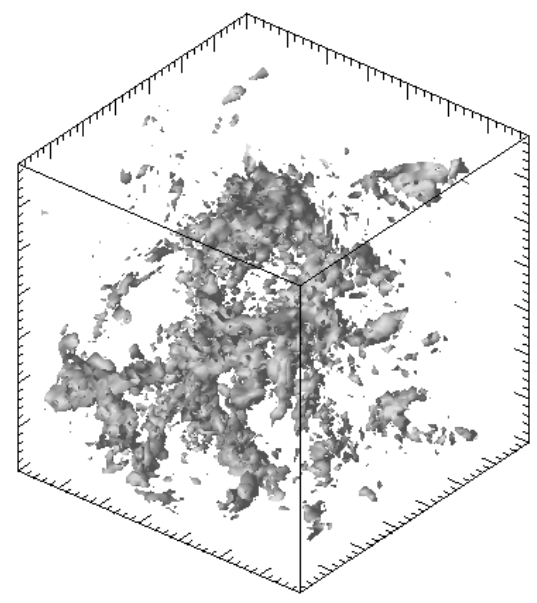

(d) $\left|\nabla \times \vec{\ell}_{i i}\right|$

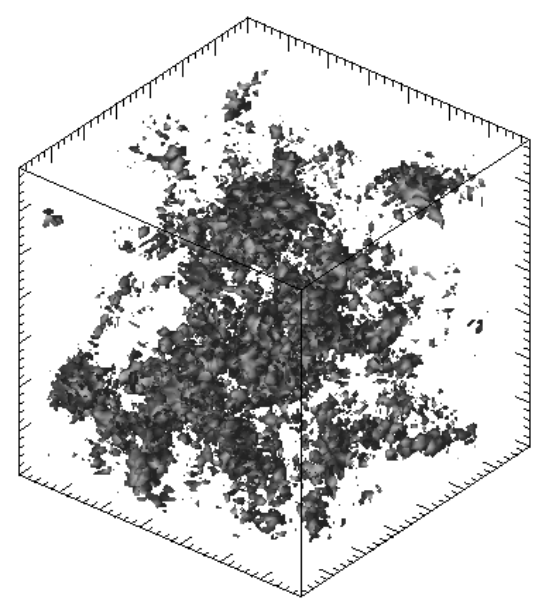

Figure 9. Components cc, ci, ic and ii of the modulus of the curl of the Lamb vector for $R_{\lambda}=140$. The isosurfaces are $3 \sigma$ where $\sigma^{\text {sub }}=587.26$ for (a), 53.96 for (b), 416.53 for (c) and 20.42 for $(\mathrm{d})$. 
(a) PDF

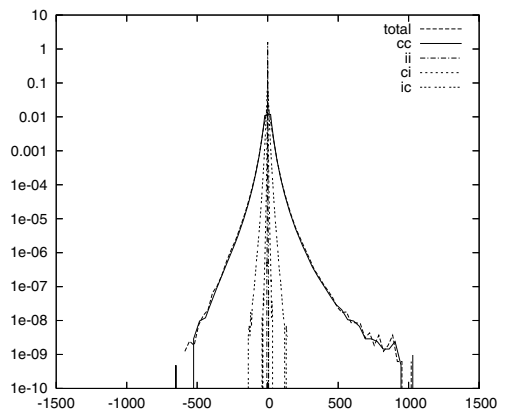

(b) spectrum

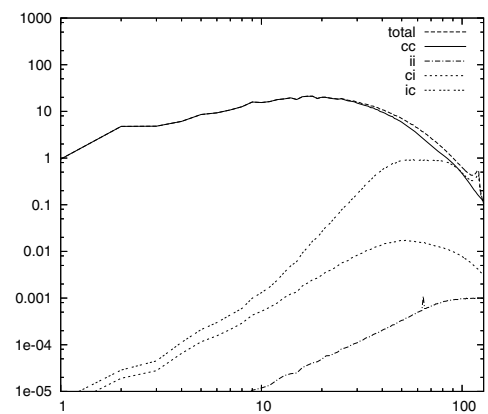

Figure 10. PDF and spectrum of the Lamb vector and its four contributions.

(a) PDF

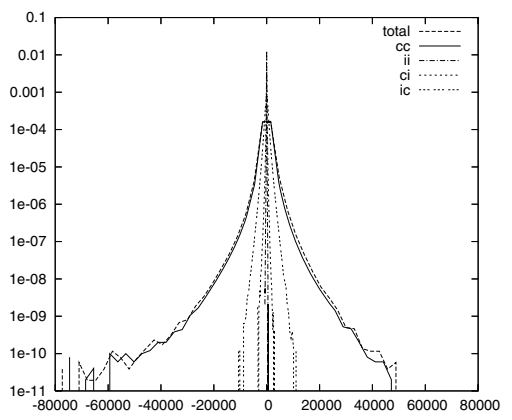

(b) spectrum

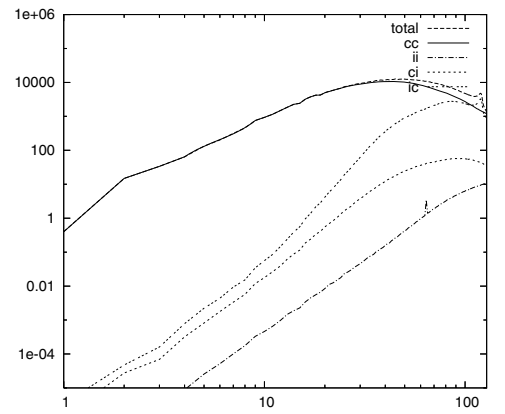

FiguRE 11. PDF and spectrum of the divergence of the Lamb vector and its four contributions.

(a) PDF

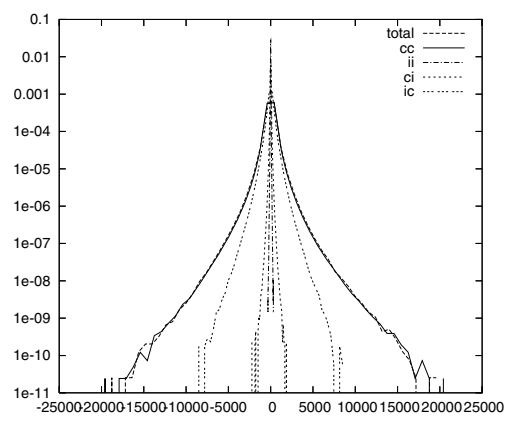

(b) spectrum

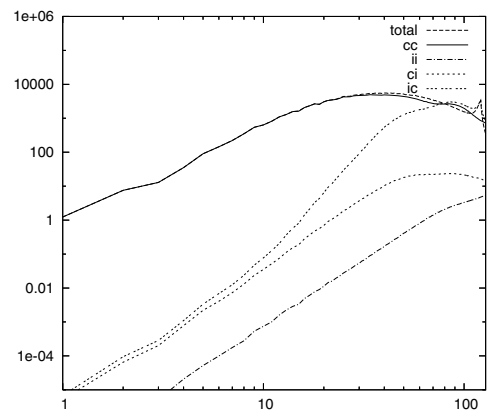

FIGURE 12. PDF and spectrum of the curl of the Lamb vector and its four contributions. 
that the coherent parts contain the structures while the incoherent parts have much weaker amplitudes and are structureless. All this is similar to the observations made for the vorticity decomposition.

We also investigated the different contributions to the Lamb vector, its divergence and its curl using the coherent and the incoherent parts of velocity and vorticity. The motivation is to study the different contributions to the nonlinear terms in the Euler equations. We found that the coherent vorticity always yields the most significant contribution.

We are very thankful to Prof. P. K. Yeung and D. Donzis from Georgia Tech for the dataset they provided us. We also thankfully acknowledge M-G. Dejean, P. Hav and P. Navaro for their helpful assistance with the computing facilities at CIRM.

\section{REFERENCES}

[1] I. Daubechies. Ten Lectures on wavelets, volume 61. CBMS-NSF Conferences in Applied Mathematics, SIAM, 1992.

[2] D. Donoho. Unconditional bases are optimal bases for data compression and statistical estimation. Appl. Comput. Harmon. Anal., 1:100-115, 1993.

[3] D. Donoho and I Johnstone. Ideal spatial adaption via wavelet shrinkage. Biometrica, 81:425-455, 1994.

[4] M. Farge. Wavelet transforms and their applications to turbulence. Ann. Rev. of Fluid Mech., 24:395-457, 1992.

[5] M. Farge, G. Pellegrino, and K. Schneider. Coherent vortex extraction in 3D turbulent flows using orthogonal wavelets. Phys. Rev. Lett., 87(5):054501, 2001.

[6] M. Farge and K. Schneider. Coherent vortex simulation (CVS), a semi-deterministic turbulence model using wavelets. Flow, Turbulence and Combustion, 66(4):393-426, 2001.

[7] M. Farge, K. Schneider, and N. Kevlahan. Non-gaussianity and coherent vortex simulation for two-dimensional turbulence using an adaptive orthonormal wavelet basis. Phys. Fluids, 11(8):2187-2201, 1999.

[8] M. Farge, K. Schneider, G. Pellegrino, A. A. Wray, and R. S. Rogallo. Coherent vortex extraction in three-dimensional homogeneous turbulence: comparision between CVS-wavelet and POD-Fourier decompositions. Phys. Fluids, 15(10):2886-2896, 2003.

[9] M.S. Howe. Theory of vortex sound. Cambridge University Press, 2003.

[10] S. Mallat. A Wavelet Tour of Signal Processing. Academic Press, 1999.

[11] O. Roussel, K. Schneider, and M. Farge. Coherent vortex extraction in 3d homogeneous turbulence : comparison between orthogonal and biorthogonal wavelet decompositions. J. of Turbulence, 6(11):1-15, 2005.

[12] K. Schneider, M. Farge, G. Pellegrino, and M. Rogers. Coherent vortex simulation of three-dimensional turbulent mixing layers using orthogonal wavelets. J. Fluid Mech., 534:39-66, 2005.

[13] P.K. Yeung, D.A. Donzis, and K.R. Sreenivasan. High-Reynolds-number simulation of turbulent mixing. Phys. of Fluids, 17:081703, 2005. 